\title{
ON NONLINEAR INTEGRAL EQUATIONS WITH DEVIATING ARGUMENTS
}

\author{
K. BALACHANDRAN AND S. ILAMARAN
}

\begin{abstract}
We prove an existence theorem for a class of nonlinear integral equations with deviating arguments.
\end{abstract}

\section{Introduction}

Several authors have studied the nonlinear Volterra integral equation with deviating arguments $[1,4,6,7,8]$. Banas [5] has proved an existence theorem for functional integral equation. Balachandran [1] has extended this theorem to a class of nonlinear Volterra integral equations with deviating arguments. Balachandran and Ilamaran $[2,3]$ established existence theorems for nonlinear integral equations with deviating arguments. In this paper we shall derive a set of sufficient conditions for the existence of a solution of a class of nonlinear integral equations with deviating arguments. The technique used in this paper is similar to the one used by Banas [5] and Balachandran and Ilamaran [2,3].

\section{Basic Assumptions}

Let $p(t)$ be a given continuous function defined on the interval $[0, \infty)$ and taking real positive values. Denote $C\left([0, \infty), p(t): R^{n}\right)$ by $C_{p}$, the set of all

Received October 15, 1991.

AMS Subject Classification: 45D05.

Key words: Existence of solution, Volterra integral equation, Deviating arguments. 
continuous functions from $[0, \infty)$ into $R^{n}$ such that

$$
\sup \{|x(t)| p(t): t \geq 0\}<\infty
$$

It has been proved [8] that $C_{p}$ forms a real Banach space with regard to the norm

$$
\|x\|=\sup \{|x(t)| p(t): t \geq 0\}
$$

If $x \in C_{p}$ then we will denote $\mathcal{W}^{T}(x, \varepsilon)$ the usual modulus of continuity of $x$ on the interval $[0, T]$ i.e.,

$$
\mathcal{W}^{T}(x, \varepsilon)=\sup \{|x(t)-x(s)|:|t-s| \leq \varepsilon, t, s \in[0, T]\}
$$

Our existence theorem is based on the following lemma.

Lemma. [6] Let $E$ be a bounded set in the space $C_{p}$. If all functions belonging to $E$ are equicontinuous on each interval $[0, T]$ and $\lim _{T \rightarrow \infty} \sup \{|x(t)| p(t)$ : $t \geq T\}=0$ uniformly with respect to $E$, then $E$ is relatively compact in $C_{p}$.

Consider the nonlinear Volterra integral equation of the form

$$
x(t)=H(t, x(t))+g\left(t, \int_{0}^{t} K(t, s, x(h(s))) d s\right)
$$

where $x, H, K$ and $g$ are $n$-vectors.

Assume the following conditions:

(i) Let $\triangle=\{(t, s): 0 \leq s \leq t<\infty\}$

The function $K: \triangle \times R^{n} \rightarrow R^{n}$ is continuous and there exists continuous functions $m: \triangle \rightarrow[0, \infty), a:[0, \infty) \rightarrow(0, \infty) b:[0, \infty) \rightarrow[0, \infty)$ such that

$$
|K(t, s, x)| \leq m(t, s)+a(t) b(s)|x|
$$

for all $(t, s) \in \triangle$ and $x \in R^{n}$.

In order to formulate other assumptions let us define

$$
L(t)=\int_{0}^{t} a(s) b(s) d s, \quad t \geq 0 .
$$


Take an arbitrary number $M>0$ and consider the space $C_{p}$ with $p(t)=$ $\left[a(t) e^{M L(t)+t}\right]^{-1}$.

(ii) there exists a constant $B>0$ such that for any $t \in[0, \infty)$ the following inequality holds

$$
\int_{0}^{t} m(t, s) d s \leq B a(t) e^{M L(t)}
$$

(iii) $H:[0, \infty) \times R^{n} \rightarrow R^{n}$ is continuous and there exists a constant $A$ such that

$$
|H(t, x(t))| \leq A|x(t)|
$$

(iv) the function $g:[0, \infty) \times R^{n} \rightarrow R^{n}$ is continuous and satisfies the Lipschitz condition

$$
|g(t, x)-g(t, y)| \leq k|x-y|
$$

where $k$ is a constant and

$$
|g(t, 0)| \leq R a(t) e^{M L(t)}
$$

(v) $h:[0, \infty) \rightarrow[0, \infty)$ is a continuous function satisfying the condition $L(h(t))-L(t) \leq N$ where $N$ is a positive constant.

(vi) $a(h(t)) / a(t) \leq(M / k)(1-A-k B-R) e^{-M N}$ where $A+k B+R<1$

\section{Existence Theorem}

Theorem. Assume that the hypotheses (i) to (vi) hold; then the equation (1) has atleast one solution $x$ in the space $C_{p}$ such that $|x(t)| \leq a(t) e^{M L(t)}$ for any $t \geq 0$.

Proof. Define a transformation $F$ in the space $C_{p}$ by

$$
(F x)(t)=H(t, x(t))+g\left(t, \int_{0}^{t} K(t, s, x(h(s))) d s\right)
$$

From our assumptions we observe that $(F x)(t)$ is continuous on the interval $[0, \infty)$. Define the set $E$ in $C_{p}$ by

$$
E=\left\{x \in C_{p}:|x(t)| \leq a(t) e^{M L(t)}\right\} .
$$


Clearly $E$ is nonempty, bounded, convex and closed in $C_{p}$. Now we prove that $F$ maps the set $E$ into itself. Take $x \in E$. Then from our assumptions we have

$$
\begin{aligned}
& |(F x)(t)| \\
\leq & |H(t, x(t))|+k \int_{0}^{t}|K(t, s, x(h(s)))| d s+|g(t, 0)| \\
\leq & A|x(t)|+k \int_{0}^{t} m(t, s) d s+k a(t) \int_{0}^{t} b(s)|x(h(s))| d s+|g(t, 0)| \\
\leq & A a(t) e^{M L(t)}+k B a(t) e^{M L(t)} \\
& +k a(t) \int_{0}^{t} b(s) a(h(s)) e^{M L(h(s))} d s+R a(t) e^{M L(t))} \\
\leq & (A+k B+R) a(t) e^{M L(t)} \\
& +M(1-A-k B-R) a(t) \int_{0}^{t} a(s) b(s) e^{M L(s)} e^{-M N} e^{M[L(h(s))-L(s)]} d s \\
\leq & (A+k B+R) a(t) e^{M L(t)}+(1-A-k B-R) a(t) \int_{0}^{t} M a(s) b(s) e^{M L(s)} d s \\
\leq & (A+k B+R) a(t) e^{M L(t)}+(1-A-k B-R) a(t) e^{M L(t)} \\
= & a(t) e^{M L(t)}
\end{aligned}
$$

which proves that $F E \subset E$.

Now we want to prove that $F$ is continuous on the set $E$. For this let us fix $\varepsilon>0$ and take $x, y \in E$ such that $\|x-y\| \leq \varepsilon$. Further take an arbitrary fixed $T>0$. In view of (i) and (iv) the functions $K(t, s, x)$ and $H(t, x)$ are uniformly continuous on

$$
[0, T] \times[0, T] \times[-r(h(t)), r(h(T))]^{n} \text { and }[0, T] \times[-r(T), r(T)]^{n}
$$

respectively, where $r(T)=\max \left\{a(s) e^{M L(s)}: s \in[0, t]\right\}$. Thus, we have for $t \in[0, T]$

$$
\begin{aligned}
|(F x)(t)-(F y)(t)| \leq & |H(t, x(t))-H(t, y(t))| \\
& +k \int_{0}^{t}|K(t, s, x(h(s)))-K(t, s, y(h(s)))| d s \\
\leq & \beta_{1}(\varepsilon)+\beta_{2}(\varepsilon)
\end{aligned}
$$


where $\beta_{i}$ are continuous functions such that $\lim _{\varepsilon \rightarrow 0} \beta_{i}(\varepsilon)=0$. Further, let us take $t \geq T$. Then we have

$$
\begin{aligned}
|(F x)(t)-(F y)(t)| & \leq|(F x)(t)|+|(F y)(t)| \\
& \leq 2 a(t) e^{M L(t)} \\
|(F x)(t)-(F y)(t)| p(t) & \leq 2 e^{-t}
\end{aligned}
$$

Hence for sufficiently large $T$ we have

$$
|(F x)(t)-(F y)(t)| p(t) \leq \varepsilon
$$

By (3) and (4) we get $F$ is continuous on the set $E$. Hence $F$ is continuous on E.

Now we prove that $F E$ is relatively compact. For every $x \in E$ we have $F x \in$ $E$ which gives $|(F x)(t)| p(t) \leq e^{-t}$. Hence $\lim _{T \rightarrow \infty} \sup \{|(F x)(t)| p(t): t \geq T\}=0$ uniformly with respect to $x \in E$.

Furthermore, let us fix $\varepsilon>0, T>0 ; t, s \in[0, T]$ such that $|t-s| \leq \varepsilon$. Then for $x \in E$, we have

$$
\begin{aligned}
& |(F x)(t)-(F x)(s)| \\
\leq & |H(t, x(t))-H(s, x(s))| \\
& +\left|g\left(t, \int_{0}^{t} K(t, u, x(h(u))) d u\right)-g\left(t, \int_{0}^{s} K(s, u, x(h(u))) d u\right)\right| \\
& +\left|g\left(t, \int_{0}^{s} K(s, u, x(h(u))) d u\right)-g\left(s, \int_{0}^{s} K(s, u, x(h(u))) d u\right)\right| \\
\leq & \mathcal{W}^{T}(H, \varepsilon)+k\left|\int_{0}^{t} K(t, u, x(h(u))) d u-\int_{0}^{s} K(s, u, x(h(u))) d u\right|
\end{aligned}
$$




$$
\begin{aligned}
& +\mathcal{W}^{T}(g, \varepsilon) \\
\leq & \mathcal{W}^{T}(H, \varepsilon)+k\left|\int_{0}^{t} \mathbb{K}(t, u, x(h(u))) d u-\int_{0}^{s} \mathbb{K}(t, u, x(h(u))) d u\right| \\
& +k\left|\int_{0}^{s} \mathbb{K}(t, u, x(h(u))) d u-\int_{0}^{s} K(s, u, x(h(u))) d u\right|+\mathcal{W}^{T}(g, \varepsilon) \\
\leq & \mathcal{W}^{T}(H, \varepsilon)+k \int_{s}^{t}|K(t, u, x(h(u)))| d u \\
& +k \int_{0}^{s}|\mathbb{K}(t, u, x(h(u)))-\mathbb{K}(s, u, x(h(u)))| d u+\mathcal{W}^{T}(g, \varepsilon) \\
\leq & \mathcal{W}^{T}(H, \varepsilon)+k \in \max \left\{m(t, u)+a(t) b(u)[p(h(u))]^{-1}: 0 \leq u \leq t \leq T\right\} \\
& +k T \mathcal{W}^{T}(K, \varepsilon)+\mathcal{W}^{T}(g, \varepsilon)
\end{aligned}
$$

which tends to zero as $\varepsilon \rightarrow 0$. Thus $F E$ is equicontinuous on $[0, T]$.

Therefore from the lemma $F E$ is relatively compact. Thus the Schauder fixed point theorem guarantees that $F$ has a fixed point $x \in \mathbb{E}$ such that $(F x)(t)=x(t)$. Hence the theorem.

\section{References}

[1] K. Balachandran, "Existence of solution for nonlinear Volterra Integral equation with deviationg argument", J. Math. Phy. Sci., 23 (1989), 201-205.

[2] K. Balachandran and S. Ilamaran, "An existence theorem for Volterra integral equation with deviating arguments", J. Appl. Math. and Stoc. Anal., Vol. 3, No. 3 (1990), 155-162.

[3] K. Balachandran and S. Ilamaran, "Existence of solution for nonlinear Volterra integral equations", Proc. Indian Acad. of Sci. (Math. Sci.), Vol 100, No. 2, August 1990, 179-184.

[4] J. Banas, "On solutions of some functional integral equations", Bubl. Inst. Math. Acad. Sinica, 12 (1984), 23-30.

[5] J. Banas, "An existence theorem for nonlinear Volterra integral equation with deviating argument", Rendiconti del Circolo Mathematico di Palermo, Series 2, 35 (1986), 82-89.

[6] J. Banas and K. Goebel, "Measure of Noncompactness in Banach Spaces", Marcel Dekkar Inc., New York (1980).

[7] T. A. Burton, "Volterra Integral and Differential Equations", Academic Press, New. York, (1983).

[8] C. Corduneanu, "Integral Equations and Stability of Feedback Systems", Academic Press, New York (1973).

Department of mathematics, Bharathiar University, Coimbatore-641 046, Tamil Nadu, India. 